\title{
Organic crystal-based THz source for complex refractive index measurements of window materials using single-shot $\mathrm{THz}$ spectroscopy
}

\author{
Lufan Du$^{1} \cdot$ Franz Roeder $^{1} \cdot$ Yun $\mathrm{Li}^{1} \cdot$ Mostafa Shalaby $^{2,3} \cdot$ Burgard Beleites $^{1} \cdot$ Falk Ronneberger $^{1} \cdot$ Amrutha Gopal $^{4,5}$
}

Received: 20 July 2021 / Accepted: 19 September 2021 / Published online: 18 October 2021

(c) The Author(s) 2021

\begin{abstract}
We employed N-benzyl-2-methyl-4-nitroaniline (BNA) crystals bonded on substrates of different thermal conductivity to generate $\mathrm{THz}$ radiation by pumping with $800 \mathrm{~nm}$ laser pulses. Crystals bonded on sapphire substrate provided four times more THz yield than glass substrate. A pyrodetector and a single-shot electro-optic (EO) diagnostic were employed for measuring the energy and temporal characterisation of the THz pulse. Systematic studies were carried out for the selection of a suitable EO crystal, which allowed accurate determination of the emitted THz spectrum from both substrates. Subsequently, the $\mathrm{THz}$ source and single-shot electro-optic detection scheme were employed to measure the complex refractive index of window materials in the $\mathrm{THz}$ range.
\end{abstract}

Keywords Terahertz (THz) generation · Organic crystals · Single-shot electro-optic (EO) detection

\section{Introduction}

The terahertz $(\mathrm{THz})$ frequency range is usually defined as the spectral regime between 0.1 and $10 \mathrm{THz}$. In the last decades $\mathrm{THz}$ radiation has attracted huge interest due to the availability of compact, broadband and tunable sources enabling real-life applications ranging from fundamental science to spectroscopic and imaging applications in industry, security and life science [1-11]. For example, terahertz waves can be employed to study carrier dynamics in semiconductor materials, imaging of biological tissues and investigation of phonon excitations

Amrutha Gopal

amrutha.gopal@uni-jena.de

Lufan Du

lufan.du@uni-jena.de

1 Institut für Optik und Quantenelektronik, Max-Wien-Platz 1, 07743 Jena, Germany

2 Swiss Terahertz Research-Zurich, Technopark, Technopark, 8005 Zurich, Switzerland

3 Park Innovaare, 5234 Villigen, Switzerland

4 Helmholtz-Institut Jena, Fröbelstieg 3, 07743 Jena, Germany

5 Institut für Optik und Quantenelektronik, Max-Wien-Platz 1, 07743 Jena, Germany and collision rates of conduction electrons in solids, etc. $[12,13]$. Furthermore, theoretical and experimental studies have shown that strong terahertz fields can be used to excite nonlinear responses in materials [14-16]. Thanks to their non-ionising nature, $\mathrm{THz}$ radiation can also be employed for biochemical analysis [17, 18]. Last decade has witnessed a tremendous growth in terahertz generation technology which in turn resulted in the development of ultra-compact sources for industrial and biological applications that reach ultrahigh peak and average powers enabling their use for studies of nonlinear processes $[2,6,19,20]$. The availability of high power $\mathrm{THz}$ sources based on organic crystals accelerated this growth thanks to their simpler experimental schemes and phase matching at commonly available laser wavelengths. N-benzyl-2-methyl-4-nitroaniline (BNA) is a novel organic crystal suitable for pumping with NIR laser pulses delivered by Titanium-Sapphire lasers. Although BNA and other organic crystals offer simple schemes to generate $\mathrm{THz}$ radiation compared to $\mathrm{LiNbO}_{3}$ crystals, the efficiency of $\mathrm{THz}$ generation is relatively low due to their low thermal capacity [6, 21-26]. Thus, different methods and techniques existing in laser technology, such as external cooling or bonding with substrate materials having high thermal conductivity, could be implemented to increase the THz yield [27, 28]. External cooling brings complexities and could be suitable 
for laser systems operating at $\mathrm{kHz}$ or higher repetition rates. However, mounting the crystals onto substrates with high thermal conductivity could reduce the heat load on them and enable a change in the optical properties resulting in increased THz yield. At first, we study and compare the performance of BNA crystals mounted on commonly employed substrate materials such as glass and sapphire and characterise the generated $\mathrm{THz}$ radiation.

Traditionally, electro-optic sampling based on the Pockels effect is employed to measure the terahertz electric field [29-31]. Here the pulsed terahertz radiation and short optical pulses enter collinearly in an electro-optic crystal. The short optical pulse is delayed in time to sample the electric field of the comparatively longer terahertz pulse, requiring a large number of measurements to trace the whole $\mathrm{THz}$ pulse. This technique is suitable for sources driven by high repetition rate laser systems. Filtering techniques are employed to improve the signal to noise ratio and shot-to-shot fluctuations. However, for $\mathrm{THz}$ sources driven by low repetition rate laser systems the above technique is not feasible. Since they can deliver high-power terahertz pulses, a noncollinear geometry can be employed which opens up the possibility of single-shot detection of the terahertz electric field using a single-shot electro-optic diagnostic [32-34]. In this scheme the terahertz pulse is incident normal to the electro-optic crystal like in the case of collinear sampling. However, a spatially extended optical probe pulse enters at an angle onto the crystal as shown in Fig. 1. This implies that different spatial components of the probe pulse arrive at different times at the crystal providing an observation time window. By adjusting the angle between the $\mathrm{THz}$ and optical beams the time window can be optimised for the measurements. Using a large area charge-coupled device (CCD) the whole $\mathrm{THz}$ pulse can be measured in a single-shot allowing faster and accurate pump-probe studies and other terahertz applications.

Combining high-power sources and single-shot $\mathrm{THz}$ detection schemes opens up a plethora of applications as mentioned earlier [5]. Similarly with increasing $\mathrm{THz}$ pulse energy it is also important to understand the optical properties of materials in the $\mathrm{THz}$ range for efficient beam control and guiding. In fact, previous studies have shown that current $\mathrm{THz}$ sources can create intense pulses which can induce optical nonlinearities in the medium as they propagate through it $[5,21,22,35,36]$. Thus, characterisation of optics and window materials for $\mathrm{THz}$ applications needs to be carried out. Until now conventional time domain spectrometers either in the reflection or transmission mode have been employed to determine the optical constants at terahertz wavelengths. Here, we will employ our high-power $\mathrm{THz}$ source to characterise different window materials using single-shot spectroscopy [36]. Then, we will compare the outcome of these studies to previous works using THz-time domain spectrometers to verify the feasibility of this method $[35,37-39]$.

\section{Experiments}

The experiments were performed at the JETI-40 (JEna Titanium-Sapphire) laser facility. It is a semi-commercial high-power laser system capable of providing peak powers up to $40 \mathrm{TW}$ for experimental studies [40]. The output from the femtosecond oscillator is amplified via chirped pulse amplification (CPA) to $1.2 \mathrm{~J}$ and re-compressed to a minimum pulse duration of $30 \mathrm{fs}$. However, for the experiments
Fig. 1 Schematic of the experimental setup for $\mathrm{THz}$ generation and detection

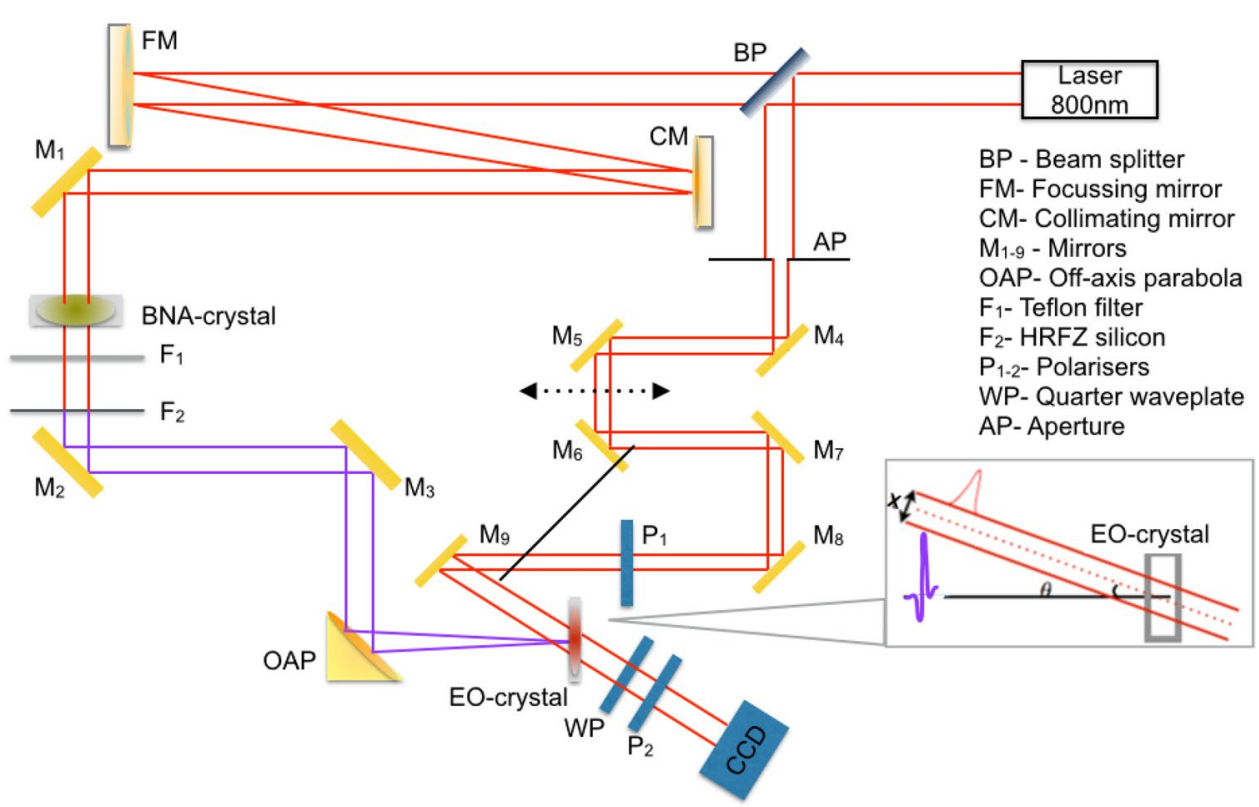


discussed here a pulse energy up to $50 \mathrm{~mJ}$ was employed. A schematic of the experimental setup is shown in Fig. 1. About $5 \%$ of the pump beam power was taken out using a beam splitter and sent to the electro-optic diagnostic for coherent detection of the $\mathrm{THz}$ pulse. The rest of the pump beam was reshaped to $2 \times 2 \mathrm{~cm}^{2}$ using reflective optics. The BNA crystal fixed on a rotary mount was exposed to the pump beam. Phase matching for optimal $\mathrm{THz}$ generation was achieved by rotating the crystal axis with respect to the laser polarisation. Initially a $600 \mu \mathrm{m}$ thick BNA crystal mounted onto a glass substrate was employed to generate $\mathrm{THz}$ radiation. The residual pump beam was filtered out at first with a Teflon sheet followed by a HRFZ ( High Resistivity Float Zone) silicon wafer. The transmitted THz beam was collected and guided using gold-coated mirrors and focused down using a f/1 parabola. At first the $\mathrm{THz}$ yield was optimised using a pyrometer fitted with a HRFZ silicon window. The measurements were carried out at 10 $\mathrm{Hz}$ repetition rate of the laser. Thereafter, the single-shot spatial encoding technique was employed to detect the $\mathrm{THz}$ electric field transient and thereby calculate the $\mathrm{THz}$ pulse spectrum [34]. The single-shot spatial encoding scheme is a coherent detection method based on the linear electro-optic effect. This means that the electric field of the THz pulse induces a variation in the refractive index of the electrooptic (EO) crystal and thereby generates an instantaneous birefringence. A co-propagating linearly polarised optical pulse experiences a change in the polarisation state due to the modulation of the refractive index of the crystal.

This phase shift experienced by the optical probe beam as it propagates through the EO crystal with a thickness $d$ can be written as

$\Phi=\frac{\omega_{o p t} d}{c}\left(n_{1}-n_{2}\right)$

where $n_{1}$ and $n_{2}$ are the slow and fast components of the refractive index. In the absence of the external field the refractive index of the medium is isotropic $\left(n_{1}=n_{2}\right)$. For optimal alignment of the optical probe and $\mathrm{THz}$ beam with respect to the crystal plane, the accumulated phase by the probe beam can be written as [30]

$\sin \Phi=\frac{\omega_{\text {opt }}}{c} n_{\text {opt }}^{3} r_{41} E_{T H z} d$

where $r_{41}$ is the electro-optic coefficient of the crystal and $n_{\text {opt }}$ is the refractive index of the EO crystal at the probe frequency $\omega_{\text {opt }}$. The above equation explains that the induced birefringence and thereby the phase change experienced by the optical probe beam is directly proportional to the $\mathrm{THz}$ electric field $\left(\mathrm{E}_{\mathrm{THz}}\right)$. The instantaneous $\mathrm{THz}$ electric field can be measured by sending a ultra-low power linearly polarised short optical probe pulse. The short optical probe pulse is delayed in time to sample the comparatively longer $\mathrm{THz}$ electric field by scanning over a longer time range, requiring longer delay ranges and sampling space, thereby suitable only for high repetition rate systems. A lock-in-detection technique can be employed to obtain high signal-to-noise ratio and improve the spectral signal [31]. For high-power THz systems, single-shot measurements based on the spatial encoding scheme are advantageous. In the spatial encoding scheme, like in the case of EO sampling, the $\mathrm{THz}$ pulse is incident normally onto the plane of the EO crystal and the optical probe beam is sent at an angle to the EO crystal. This results in different arrival times for different spatial components of the probe beam as shown in the inset of Fig. 1. In other words, by choosing the optimal crystal width, probe beam width ( $\mathrm{x}$ ) and incident angle $(\theta)$ for the probe beam, a suitable observation time window $\left(T=\frac{x}{c} \tan \theta\right)$ which allows the full readout of the temporal evolution of the terahertz pulse in a single-shot can be obtained. The variation in the spatial intensity profile of the probe pulse can be recorded using a suitable imaging scheme equipped with a high dynamic range charge-coupled device (CCD).

The selection of suitable EO-crystal parameters is an important task for the optimal detection of $\mathrm{THz}$ radiation. The bandwidth and signal strength of the detection depend on the thickness and material of the EO crystal. From Eq. 2 it could be interpreted that thicker crystals and a higher value of the refractive index at the probe wavelength can induce a larger phase shift. However, the term $r_{41}$ together with the thickness of the crystal defines the bandwidth of detection. For optimum EO signal, the phase-matching condition [30] between the two pulses has to be met, $\Delta k=\frac{1}{\lambda}\left(n_{p h_{T H z}}-n_{g_{\text {opt }}}\right)=0$. In particular, the group velocity of the probe pulse should match closely with the phase velocity of the THz pulse inside the EO crystal.

We evaluated numerically the phase-matching condition for the $\mathrm{ZnTe}$ and $\mathrm{GaP}$ crystals prior to analysing their electro-optic response functions. For both crystals the complex refractive indices were obtained by evaluating the complex dielectric function $\epsilon(f)=\epsilon_{e l}+\sum_{j} \frac{S_{j} f_{j}^{2}}{f_{j}^{2}-f^{2}-i \Gamma_{j} f}$. Here the first and second terms correspond to the contributions from bound electrons and lattice oscillations, respectively [41-44]. It can be seen in Fig. 2 that the requirements for better phase matching and lower reflection losses are met by $\mathrm{ZnTe}$, thereby placing it at an advantageous position compared to GaP provided the $\mathrm{THz}$ spectrum only contains spectral components below 4.5 THz. Although the phase-matching condition for a $800 \mathrm{~nm}$ probe pulse and $\mathrm{THz}$ beam in GaP crystal is less optimal, due to their higher frequency of the transverse optical (TO) lattice oscillation resonances, thin $\mathrm{GaP}$ crystals can detect $\mathrm{THz}$ frequencies up to $10.8 \mathrm{THz}$ compared to $\mathrm{ZnTe}$, which possesses a TO resonance at $4.5 \mathrm{THz}$. Consequently, we 
Fig. 2 a Optical refractive indices at the probe wavelength and b terahertz refractive indices for the two crystals employed in this study

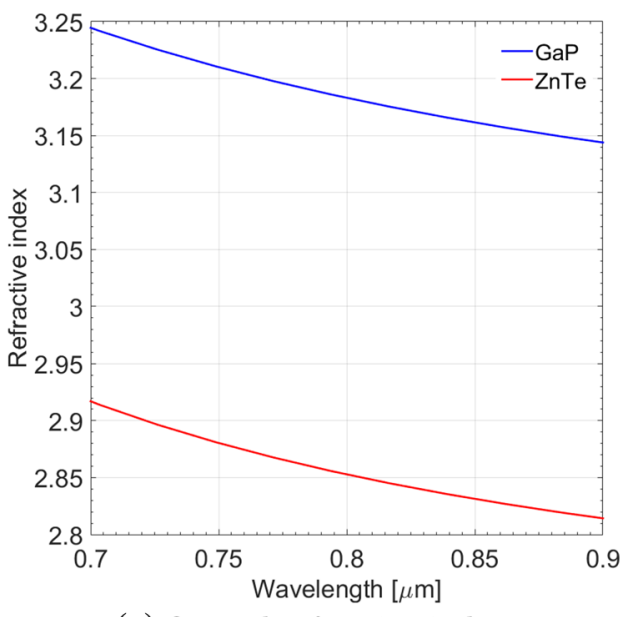

(a) Optical refractive index

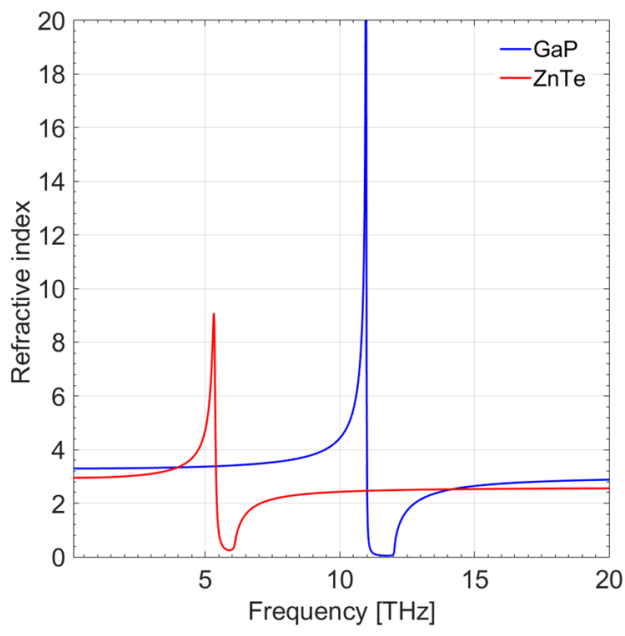

(b) $\mathrm{THz}$ refractive index evaluated and compared the crystal response functions for different thicknesses of the two crystals by taking into consideration the effective phase mismatch between the probe and $\mathrm{THz}$ pulse, absorption and transmission of $\mathrm{THz}$ radiation in the crystal. The outcome of this study is presented in Fig. 3a where solid and dotted lines represent $\mathrm{GaP}$ and ZnTe crystals, respectively. In both cases, for thinner crystals the absorption near the lattice oscillation
Fig. 3 a Plots of the EO response function for $\mathrm{ZnTe}$ and $\mathrm{GaP}$ crystals of thicknesses from 100-500 $\mu \mathrm{m}$. b Phase delay experienced by the optical probe pulse for different EO crystals. c Time domain signals of the $\mathrm{THz}$ pulse generated by BNA crystals measured with different EO crystals and $\mathbf{d}$ their corresponding spectra obtained by the Fourier transformation of $(\mathbf{c})$

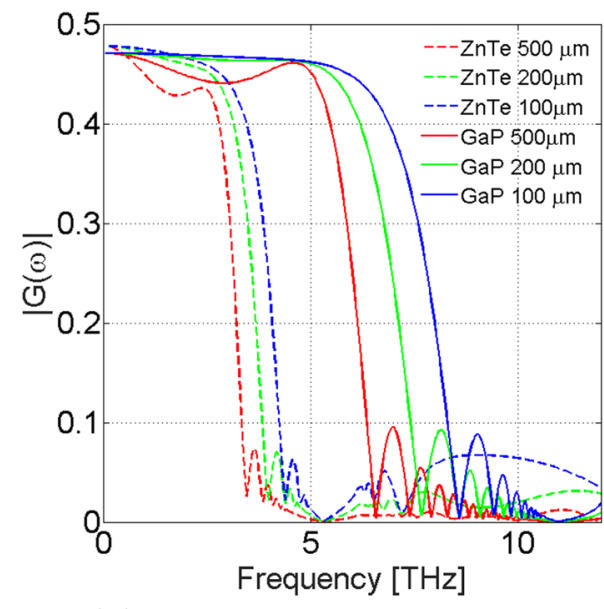

(a) EO response function

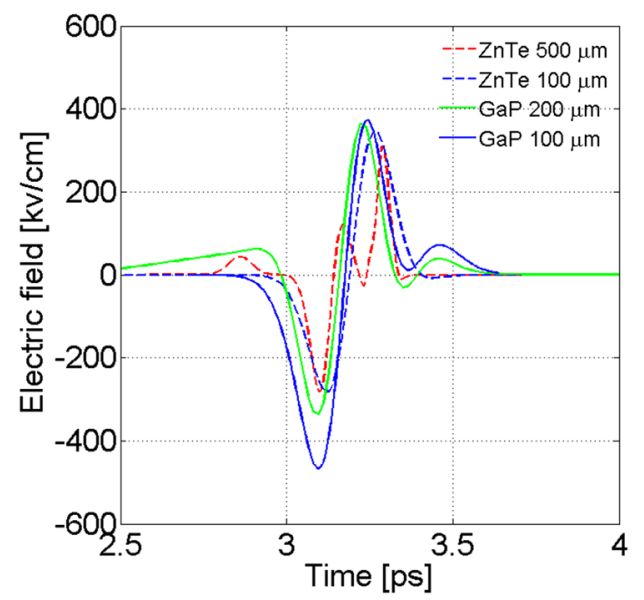

(c) Time domain

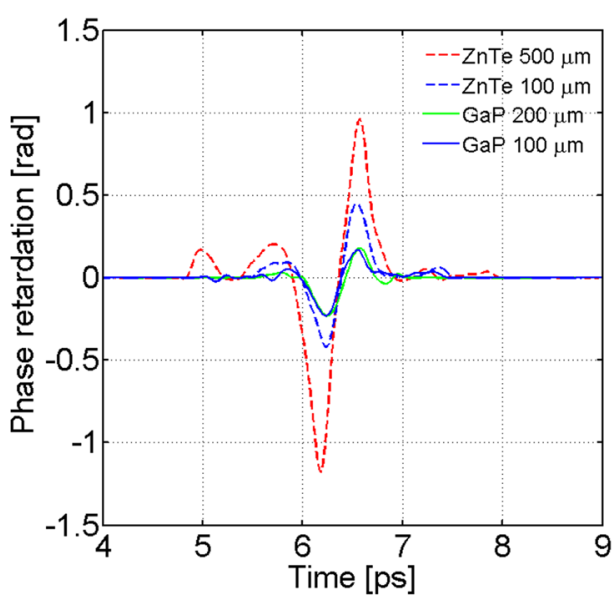

(b) Phase retardation

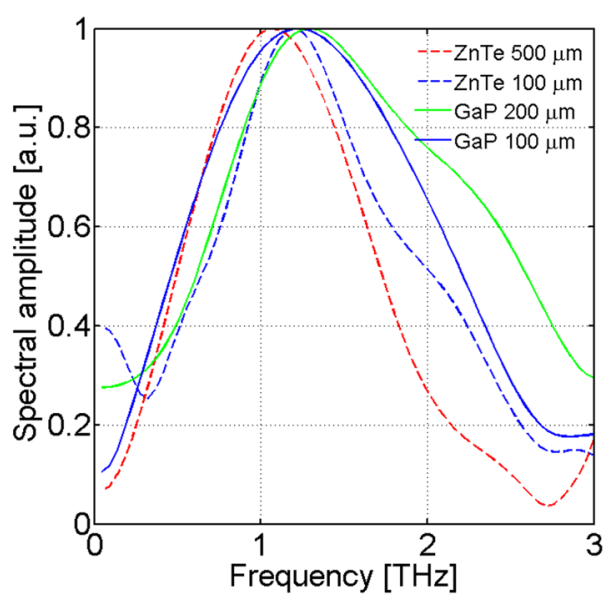

(d) Frequency domain 
frequency limits the bandwidth of detection. In the experiments, we tested the performance of both $\mathrm{GaP}$ and $\mathrm{ZnTe}$ crystals with various thicknesses.

\section{Results}

\subsection{Selection of a suitable EO crystal}

In order to select a suitable EO crystal for our studies, we employed 100 and $500 \mu \mathrm{m}$ thick ZnTe crystals as well as 100 and $200 \mu \mathrm{m}$ thick GaP crystals. THz radiation was generated with a $600 \mu \mathrm{m}$ BNA crystal mounted on a glass substrate. The phase retardation measured by the singleshot EO detection scheme is presented in Fig. 3b. The phase retardation experienced by the probe pulse is the highest for the ZnTe crystal with $500 \mu \mathrm{m}$ thickness, which in fact shows an over rotation of the phase retardation ( $\sin \Phi \geq 1$ ). Meanwhile, employing a thinner ZnTe crystal or a GaP crystal with a lower electro-optic coefficient reduces saturation effects and the measured phase retardation has a magnitude less than 1 .

For the same thickness, ZnTe offers a factor of three higher signal than GaP. Considering the fact that $\mathrm{ZnTe}$ $\left(r_{41}=4.1 \mathrm{p} \mathrm{m} / \mathrm{V}\right)$ has an electro-optic coefficient four times higher than $\mathrm{GaP}\left(r_{41}=0.88 \mathrm{pm} / \mathrm{V}\right)$ and a lower refractive index, the detected phase retardation by the two crystals agrees with the expected value. Next, the time domain signals were calculated using Eq. 2 while taking into consideration the noncollinear geometry and the outcome is presented in Fig. 3c. Despite the different optical properties, the calculated $\mathrm{THz}$ field strengths are comparable. In the case of $500 \mu \mathrm{m} \mathrm{ZnTe}$, the plot shows broadening or multiple peaks due to over rotation. Meanwhile, GaP measurements provide comparatively higher field strengths thus giving a more accurate estimate of the field strength. Subsequently, taking the Fourier transformation of the time domain signals provided the spectra of the THz pulse presented in Fig. 3d. It is evident that, despite the difference in detection bandwidths, the spectra remain centred around $1.2 \mathrm{THz}$ with frequency components extending up to $3 \mathrm{THz}$. Therefore, we selected a $100 \mu \mathrm{m} \mathrm{ZnTe}$ crystal over GaP for the subsequent studies due to the former's higher value of the electro-optic coefficient. Slight variation in the appearance of the peaks in the time domain signal can be observed which could be due to the imperfect spatial positioning of the EO crystal while placing it on the crystal holder. Due to the noncollinear geometry even small variation in the crystal thicknesses and crystal positioning can cause this temporal shift, while the broadening of the pulses in the crystals in the time domain, could lead to modifications in the spectra that are not represented in the crystal transfer functions.

\subsection{THz source and characterisation}

In our previous work, BNA crystals bonded onto glass substrates were employed to generate few micro-Joule $\mathrm{THz}$ pulses with a laser to $\mathrm{THz}$ conversion efficiency of $0.2 \%$ at $10 \mathrm{~Hz}$ repetition rate [25]. It was also found that the maximum laser fluence for optimal operation is $8 \mathrm{~mJ} / \mathrm{cm}^{2}$ and the $\mathrm{THz}$ yield saturates at fluences above $10.5 \mathrm{~mJ} / \mathrm{cm}^{2}$. We think that this saturation effect could be attributed to the heating of the crystal and the subsequent change in the refractive index that leads to a phase mismatch between the pump and the generated $\mathrm{THz}$ radiation resulting in the reduction in the effective nonlinear interaction length in the crystal. This suggests that a higher heat dissipation from the crystal could allow increased laser fluence and thereby enabling higher $\mathrm{THz}$ yield. Indeed it is well understood in laser physics that bonding crystals onto materials with higher thermal conductivity can dissipate the heat and reduce the heat load $[45,46]$. Hence, we tested and compared glass and sapphire as substrate materials for BNA [26]. BNA crystals of $5 \times 7 \mathrm{~mm}^{2}$ size and $600 \mu \mathrm{m}$ thickness were mounted onto two inch diameter glass and sapphire substrates of one millimetre thickness. The substrates were exposed to the laser radiation and the generated $\mathrm{THz}$ radiation propagated freely into air. The outcome of this study is presented in Fig. 4. It can be seen that for the same input laser fluence, a BNA crystal bonded onto a sapphire substrate generates more than four times higher THz yield compared to glass substrate (Fig. 4a).

Here, the incident laser fluence is adjusted for the Fresnel reflection losses by the two substrates. In the case of a glass substrate the $\mathrm{THz}$ yield increased quadratically and tends to saturate at higher fluences [25], while BNA on sapphire substrate does not show any sign of saturation in the inspected pump fluence regime. Increased thermal load at higher fluences caused browning of the BNA crystal at $\sim 10.5 \mathrm{~mJ} / \mathrm{cm}^{2}$ for the glass substrate and $\sim 14 \mathrm{~mJ} / \mathrm{cm}^{2}$ for the sapphire substrate. Subsequently, we looked at the time domain signal of the THz pulses and the outcome of these measurements are shown in Fig. 4b. The peak to peak electric field value for the sapphire substrate is more than 2 times higher in comparison to the glass substrate, while the respective temporal durations of $430 \pm 11 \mathrm{fs}$ and $450 \pm 11 \mathrm{fs}$ are comparable.

The frequency spectra of the $\mathrm{THz}$ pulses are obtained by the Fourier transformation of time domain signals. The spectra presented in Fig. 4c show no significant difference due to the substrate material. In Fig. 5, the EO measurements of the electric field as a function of laser fluence for the two substrate materials are presented. Similar to the outcome of the pyrodetector measurements, an increase in the $\mathrm{THz}$ field with respect to the incident laser fluence is evident. Calculating peak-peak fields for both substrates we can observe a linear increase with laser fluence. In the case of the glass substrate the observed conversion efficiency is $\sim 0.2 \%$ while 


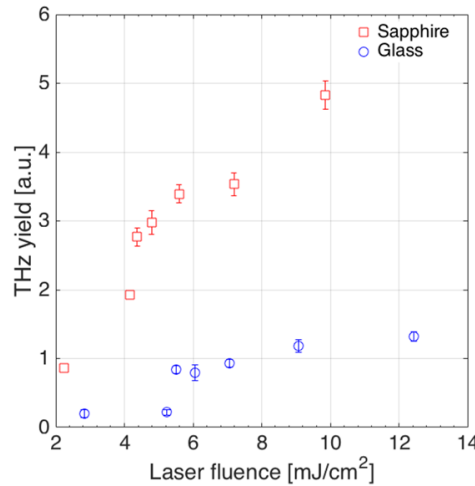

(a) $\mathrm{THz}$ yield recorded with a pyrometer

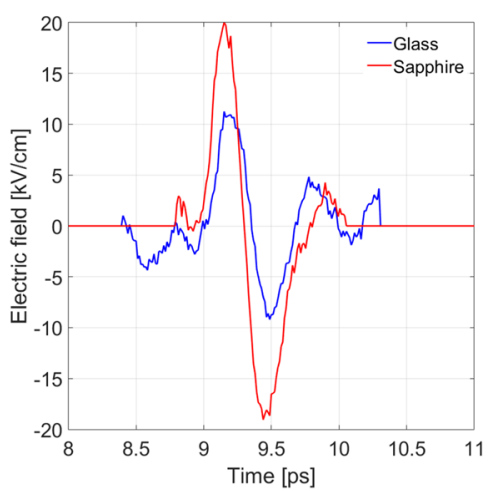

(b) Temporal waveform

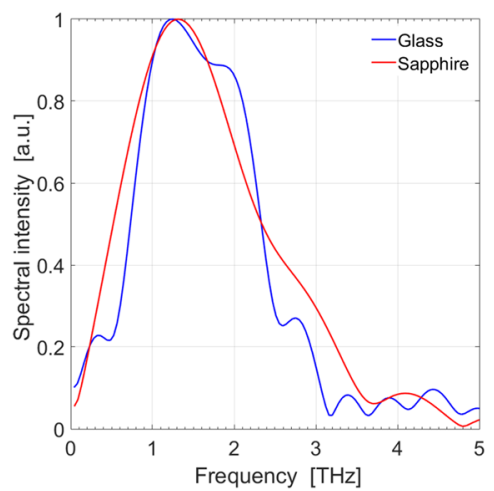

(c) Spectra of the terahertz pulses

Fig. 4 a Results of terahertz yield measurements from pyrodetector. b THz electric field recorded with the EO detection scheme and $\mathbf{c}$ the spectral content of the THz pulse calculated from the Fourier transform of the time domain data

Fig. 5 Results of EO measurements for glass (a) and sapphire (b) substrates for various incident laser fluences

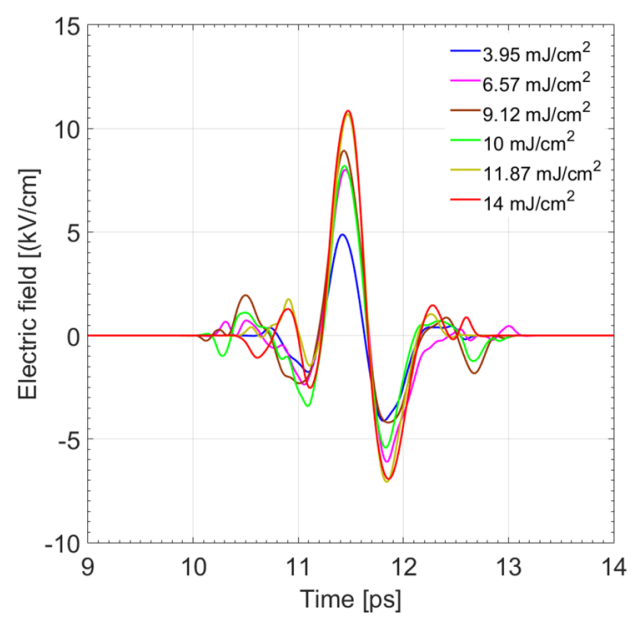

(a) Glass substrate

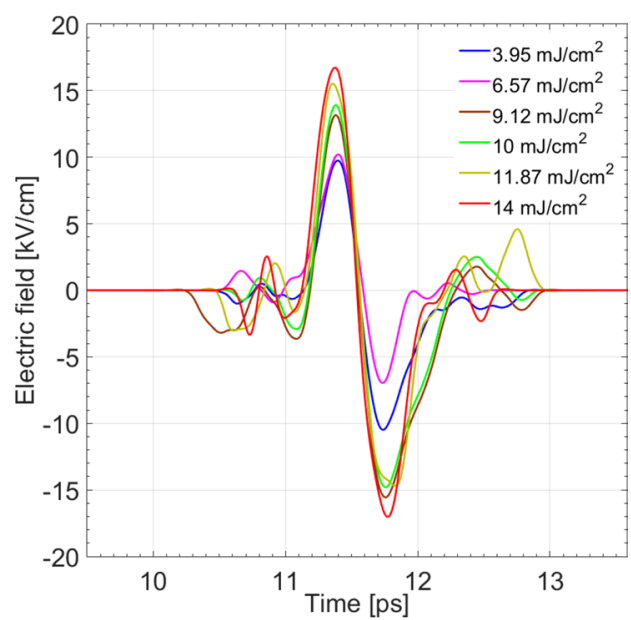

(b) Sapphire substrate for the sapphire substrate it is $\sim 0.8 \%$. This swift increase in $\mathrm{THz}$ yield could be understood based on the thermo-optical effect. Although the time interval between consecutive laser pulses is $100 \mathrm{~ms}$, due to the peak power of the laser radiation, an instantaneous increase in the crystal temperature occurs. This in turn can vary the refractive index and thereby the terahertz generation process. BNA is a crystal belonging to the aniline family which are known to have a low melting point around $100{ }^{\circ} \mathrm{C}$ while its thermal and optical properties are not yet fully studied [6,47-52]. Due to its high value of thermal conductivity a sapphire $(32 \mathrm{~W} / \mathrm{K} \mathrm{m})$ substrate dissipates the heat faster than glass $(1 \mathrm{~W} / \mathrm{K} \mathrm{m})$ and reduces the refractive index variation due to a rise in temperature. This may suggest that the BNA crystal temperature remains low and thereby at a constant refractive index for sapphire bonding, while in the case of glass the crystal temperature rises and the refractive index mismatch between the pump and the $\mathrm{THz}$ pulse increases. By measuring their thermal expansion coefficient and thermo-optic coefficient it would be easier to choose a suitable substrate material for heat dissipation. A detailed study on the thermal properties of BNA crystal is a subject of an independent study.

Next, we discuss the application of our $\mathrm{THz}$ source and single-shot $\mathrm{THz}$ detection scheme for the characterisation of common window materials. With increasing pulse energies, organic crystal-based $\mathrm{THz}$ sources can be employed for time resolved pump-probe studies [5]. Many of these experiments are conducted in restricted environments that require $\mathrm{THz}$ pulses propagating through window materials to arrive at the physical process under investigation. We tested windows made of high resistive float zone (HRFZ) silicon $(3.04 \mathrm{~mm})$, diamond $(500 \mu \mathrm{m})$, sapphire $(5 \mathrm{~mm})$, polycarbonate $(5 \mathrm{~mm})$, TPX (6.1 mm), Teflon $(3.3 \mathrm{~mm})$ and PMMA $(500 \mu \mathrm{m})$, respectively. The transverse size of the THz beam was comparable to the BNA crystal dimensions while the windows had a diameter of $5 \mathrm{~cm}$ or more. 


\subsection{THz spectroscopy of window materials}

The free standing windows were placed in the collimated $\mathrm{THz}$ beam path and the transmitted $\mathrm{THz}$ pulse has been recorded with EO detection. Based on the results from the EO measurements presented in the previous section, we chose a $500 \mu \mathrm{m} \mathrm{ZnTe}$ crystal as our $\mathrm{THz}$ detector while considering the reduction in the $\mathrm{THz}$ signal due to the window materials. In Fig. 6 the time domain signal (a) and the corresponding spectra (b) are shown. Upon insertion of the sample, the THz pulse is delayed from the observation time window in its absence. This requires the temporal delay of the probe pulse to be adjusted such that the $\mathrm{THz}$ pulse appears at the observation window of the reference pulse. The time domain signal also provides a first insight into the suitability of the window material for applications. Fresnel reflection losses cause a decrease in the amplitude of the detected signal while strong absorption and dispersion lead to broadening of the time domain signal. Although none of the samples showed any strong absorption or dispersion except PMMA, it can be easily said that teflon and TPX are promising candidates in terms of high transmissivity and economy.

For easy alignment of the optical setup TPX and Tsurupica are also candidates due to their transparency in the optical spectral regime. From the spectra of the time domain signal presented in Fig. 6b, we can understand that none of the samples except PMMA shows any signatures of absorption. For other samples, the decrease in the signal is mainly due to Fresnel losses. Despite lower thickness compared to TPX and other polymers, PMMA shows strong absorption and dispersion.

We further analysed the data by extracting the spectrally resolved refractive index and absorption coefficient from the spectral amplitudes. The Fourier transform of the respective time domain signals provided the complex frequency spectra of the terahertz pulse with $\left(\tilde{E}_{\text {samp }}(\omega)\right)$ and without $\left(\tilde{E}_{r e f}(\omega)\right)$ the presence of the sample. From the complex amplitude spectra, the spectrally resolved refractive index $\left(n_{s}(\omega)\right)$ and absorption coefficient $\left(\alpha_{s}(\omega)\right)$ were calculated using the magnitude and phase of the spectral ratios $[12,31$, 53]. The spectrally resolved refractive index and absorption coefficient of the common window materials employed in THz systems are presented in Figs. 7 and 8. The error bars show the shot-to-shot fluctuation of the laser fluence. Comparing our results obtained with a single-shot $\mathrm{THz}$ detection scheme to reference data recorded using standard THzTDS employing EO sampling technique, we can see that the results agree within two decimal places [35, 37-39].

We can see that the spectrally resolved refractive indices of polymer windows are all below 1.6 while the refractive indices of crystalline materials are higher. For both silicon and sapphire it is close to 3.4 and around 2.2 for diamond. Also no significant change over the entire spectral range is present except for the PMMA sample. Although crystalline materials extend better thermal stability for vacuum applications, their high values of the refractive indices cause reflection losses. This could be partially solved by parylene coating of the surfaces [39] or via diffraction methods [54].

The absorption coefficients calculated from the spectral amplitudes are presented in Fig. 8. Error bars represent the shot-to-shot fluctuation arising from laser fluence. The extracted values of $\alpha_{s}(\omega)$ are well below $5 \mathrm{~cm}^{-1}$ except for PMMA. Absorption is the lowest for diamond sample while PMMA exhibits the highest absorption value of 11 $\mathrm{cm}^{-1}$ at $0.8 \mathrm{THz}$. The data for refractive index and absorption coefficient of window materials calculated from the single-shot $\mathrm{THz}$ detection scheme agrees closely with the data obtained from a traditional time-domain spectrometer $[55,56]$. Large error bars in the measurements could be attributed to the shot-to-shot fluctuations in the laser energy associated with high power laser systems, which directly influence the absorption measurements. Using the complex value of the refractive index we also estimated the complex dielectric constant of the materials using the relations $\epsilon^{\prime}=[n(\omega)]^{2}-[\kappa(\omega)]^{2}$ and $\epsilon^{\prime \prime}=2 n(\omega) \kappa(\omega)$, where
Fig. 6 a A comparison of the transmitted THz pulses through window materials obtained from the EO detection-based single-shot spectrometer $\mathbf{b}$ and the corresponding transmitted spectra

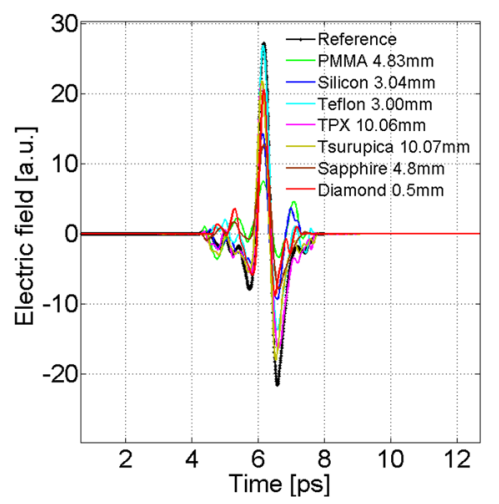

(a) Time domain signal from single-shot EO detection

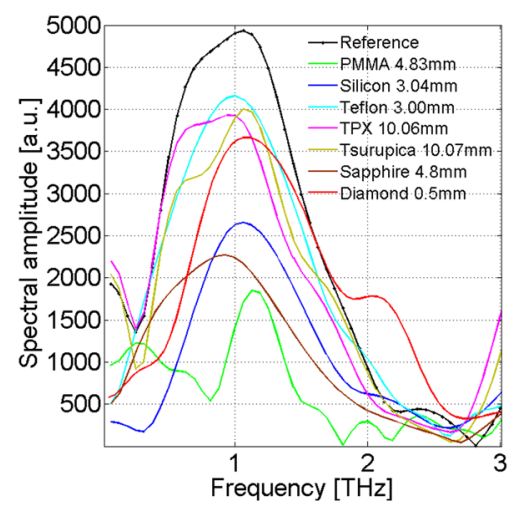

(b) Spectra of the transmitted $\mathrm{THz}$ pulse 
Fig. 7 Spectrally resolved refractive indices calculated from the Fourier transform of the single-shot time domain signal
Fig. 8 Spectrally resolved absorption coefficient calculated from the Fourier transform of the single-shot time domain signal

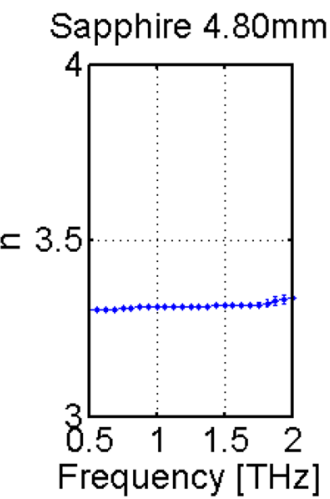

(a)

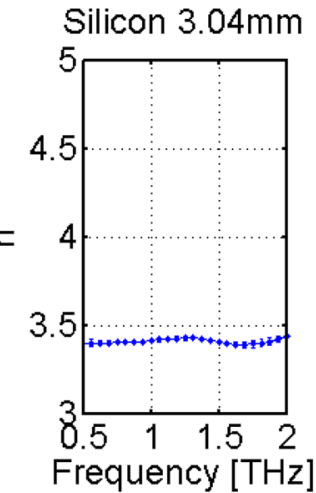

(b)

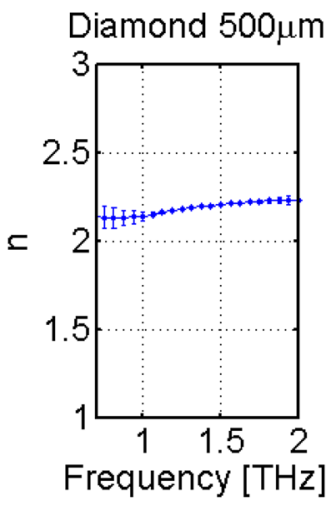

(c)

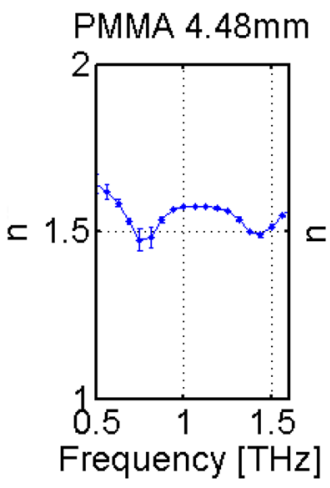

(d)

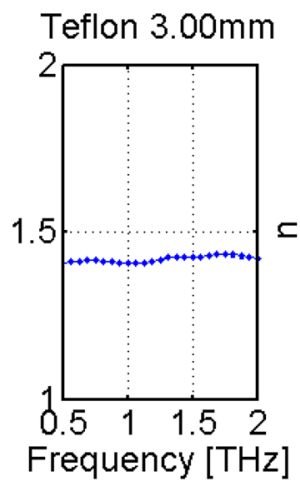

(e)
Tsurupica $10.07 \mathrm{~mm}$

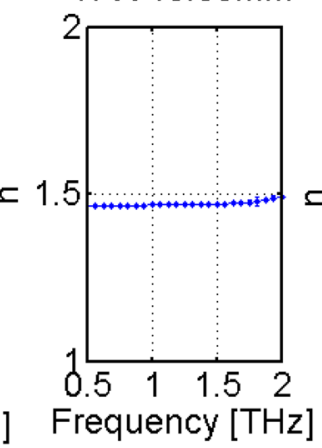

(f)

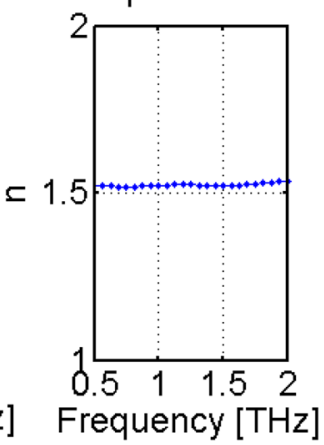

(g)

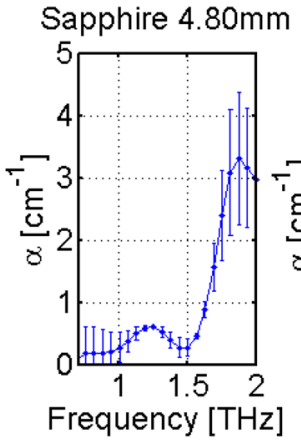

(a)

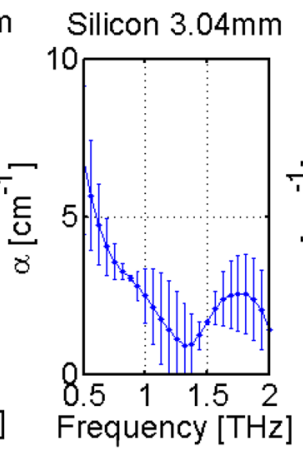

(b)

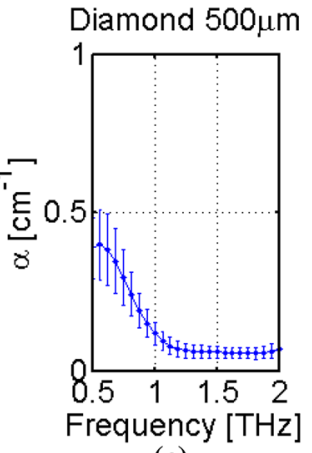

(c)

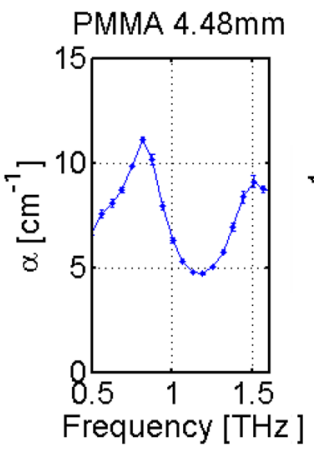

(d)

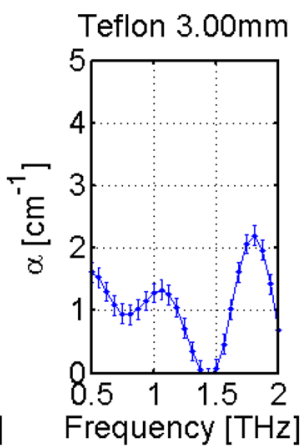

(e)

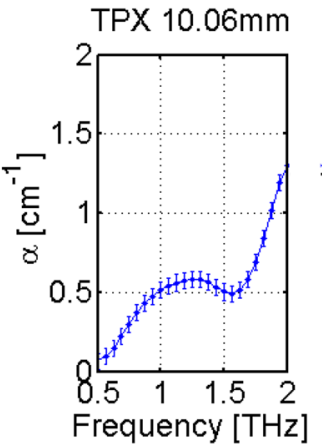

(f)

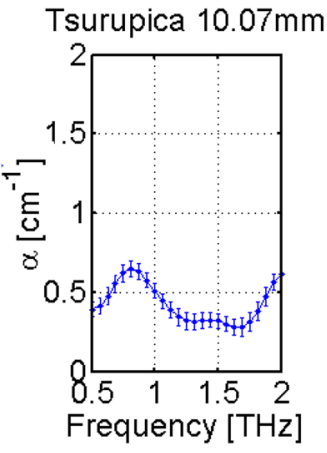

(g) 
$\kappa(\omega)=\alpha(\omega) c /(2 \omega)$. The results are presented in Fig. 9. We can see that the real part of the dielectric constant does not change significantly for most samples except PMMA as the contribution from the absorption term is negligible. The imaginary part of the dielectric constant does show some variation with frequency, albeit having a very low value. In the case of silicon and diamond it decreases with increasing frequency while sapphire shows an opposite trend. The very low value for the imaginary term of the dielectric constant implies that the main losses in the investigated samples arise from the bound carriers which contribute to the real part of the dielectric constant. This conclusion is also supported by estimation of the dielectric loss $\left(\tan \delta=\epsilon^{\prime} / \epsilon^{\prime \prime}\right)$ for the samples. Among the crystalline materials, silicon has the highest value of dielectric loss of $0.8 \times 10^{-4}$ and in the case of polymer samples, PMMA experiences the highest loss of $5 \times 10^{-4}$.

\section{Summary}

Terahertz radiation generated from BNA crystals mounted on glass substrate pumped with NIR laser pulse was detected and characterised using a single-shot electrooptic detection scheme. EO crystals of different detection bandwidths were employed to corroborate the $\mathrm{THz}$ spectra. Based on these studies a suitable EO crystal was chosen for the comparative study using BNA crystals mounted on substrates of different thermal conductivity. A sapphire substrate is found to increase the damage threshold, thermal stability and also the laser to $\mathrm{THz}$ conversion efficiency by a factor of 4 . Subsequently, the high-power $\mathrm{THz}$ source and single-shot $\mathrm{THz}$ detection scheme were employed for the characterisation of common window materials. Time domain signals for reference and samples were recorded. Afterwards, the Fourier transformation of the time domain signal was employed to obtain the amplitude and phase information of spectrum, from which the spectrally resolved refractive index and absorption coefficient of the samples were calculated. Analysis showed significant absorption in the spectrum for PMMA samples. Meanwhile, there is almost no attenuation or absorption in the observed spectral regime for Teflon and TPX, projecting them to be the best choice for a window material. However, for this conclusion we have not considered the variation in the refractive index due to a possible temperature rise in high vacuum environments. In this case crystalline materials like diamond or sapphire would be a better candidate. Comparing the spectrally resolved dielectric properties measured using single-shot $\mathrm{THz}$ detection scheme are close to the reference data obtained using standard THz- TDS employing the EO sampling technique. The results agree within two decimal places, thus demonstrating the suitability of single-shot spectroscopy for material characterisation. Although a single-shot scheme lacks the superior signal to noise ratio extended by the traditional time domain spectroscopy systems, with the availability of organic crystal-based sources, the single-shot scheme is suitable for pump-probe and nonlinearity studies.

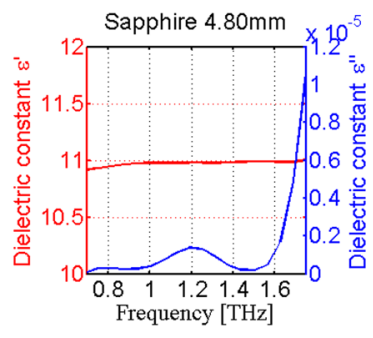

(a)

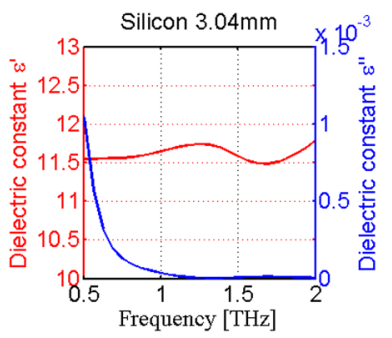

(b)

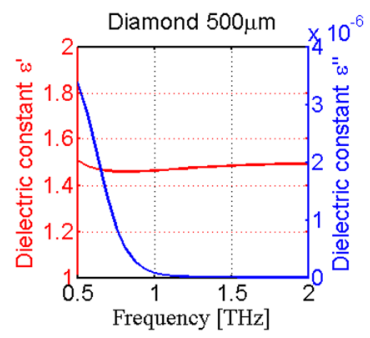

(c)

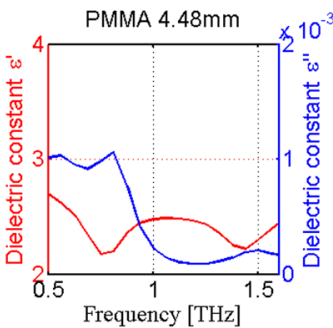

(d)

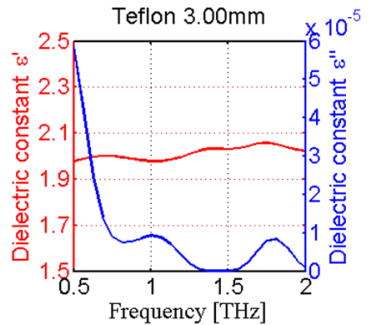

(e)

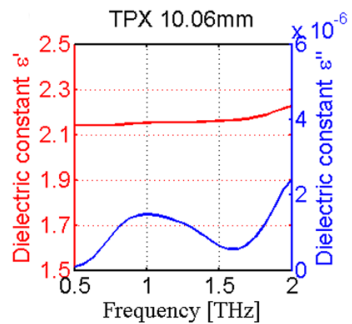

(f)

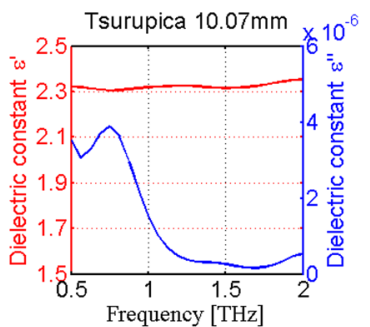

(g)

Fig. 9 The real and imaginary components of the dielectric constants for the window materials 
Funding Open Access funding enabled and organized by Projekt DEAL.

Open Access This article is licensed under a Creative Commons Attribution 4.0 International License, which permits use, sharing, adaptation, distribution and reproduction in any medium or format, as long as you give appropriate credit to the original author(s) and the source, provide a link to the Creative Commons licence, and indicate if changes were made. The images or other third party material in this article are included in the article's Creative Commons licence, unless indicated otherwise in a credit line to the material. If material is not included in the article's Creative Commons licence and your intended use is not permitted by statutory regulation or exceeds the permitted use, you will need to obtain permission directly from the copyright holder. To view a copy of this licence, visit http://creativecommons.org/licenses/by/4.0/.

\section{References}

1. J. Shi, Z. Li, D.K. Sang, Y. Xiang, J. Li, S. Zhang, H. Zhang, $\mathrm{THz}$ photonics in two dimensional materials and metamaterials: properties, devices and prospects. J. Mater. Chem. 6, 1291-1306 (2018)

2. J.A. Fülöp, S. Tzortzakis, T. Kampfrath, Laser-driven strongfield terahertz sources. Adv. Opt. Mater. 8, 1900681 (2020)

3. S.S. Dhillon, M.S. Vitiello, E.H. Linfield, A.G. Davies, M.C. Hoffmann, J. Booske, C. Paoloni, M. Gensch, P. Weightman, G.P. Williams, E. Castro-Camus, D.R. Cumming, F. Simoens, I. Escorcia-Carranza, J. Grant, S. Lucyszyn, M. KuwataGonokami, K. Konishi, M. Koch, C.A. Schmuttenmaer, T.L. Cocker, R. Huber, A.G. Markelz, Z.D. Taylor, V.P. Wallace, J. Axel Zeitler, J. Sibik, T.M. Korter, B. Ellison, S. Rea, P. Goldsmith, K.B. Cooper, R. Appleby, D. Pardo, P.G. Huggard, V. Krozer, H. Shams, M. Fice, C. Renaud, A. Seeds, A. Stïh, M. Naftaly, N. Ridler, R. Clarke, J.E. Cunningham, M.B. Johnston, The 2017 terahertz science and technology roadmap. J. Phys. D: Appl. Phys. 50, 043001 (2017)

4. S. Du, K. Yoshida, Y. Zhang, I. Hamada, K. Hirakawa, Terahertz dynamics of electron-vibron coupling in single molecules with tunable electrostatic potential. Nat. Photon. 12, 608-613 (2018)

5. T. Kampfrath, K. Tanaka, K. A. Nelson, Resonant and nonresonant control over matter and light by intense terahertz transients. Nat. Photon. 7, 680-690 (2013)

6. M. Jazbinsek, U. Puc, A. Abina, A. Zidansek, Organic crystals for THz photonics. Appl. Sci. 9, 882 (2019)

7. Y.K. Srivastava, A. Chaturvedi, M. Manjappa, A. Kumar, G. Dayal, C. Kloc, R. Singh, $\mathrm{MoS}_{2}$ for ultrafast all-optical switching and modulation of $\mathrm{THz}$ Fano metaphotonic devices. Adv. Opt. Mater. 5(23), 1700762 (2017)

8. B. Gerislioglu, A. Ahmadivand, N. Pala, Tunable plasmonic toroidal terahertz metamodulator. Phys. Rev. B 97(16), 161405 (2018)

9. G. Scalari, C. Maissen, D. Turčinková, D. Hagenmüller, S. De Liberato, C. Ciuti, C. Reichl, D. Schuh, W. Wegscheider, M. Beck, J. Faist, Ultrastrong coupling of the cyclotron transition of a $2 \mathrm{D}$ electron gas to a $\mathrm{THz}$ metamaterial. Science $\mathbf{3 3 5}(6074)$, 1323-1326 (2012)

10. A. Bayer, M. Pozimski, S. Schambeck, D. Schuh, R. Huber, D. Bougeard, C. Lange, Terahertz light-matter interaction beyond unity coupling strength. Nano Lett. 17(10), 6340-6344 (2017)

11. A. Ahmadivand, B. Gerislioglu, R. Ahuja, Y.K. Mishra, Terahertz plasmonics: the rise of toroidal metadevices towards immunobiosensings. Mater. Today 32, 108-130 (2020)
12. P.U. Jepsen, D.G. Cooke, M. Koch, Terahertz spectroscopy and imaging-modern techniques and applications. Laser Photon. Rev. 5(1), 124-166 (2011)

13. J. Hebling, K.L. Yeh, M.C. Hoffmann, K.A. Nelson, High-power $\mathrm{THz}$ generation, $\mathrm{THz}$ nonlinear optics, and $\mathrm{THz}$ nonlinear spectroscopy. IEEE J. Sel. Top. Quantum Electron. 14, 345-353 (2008)

14. D. Nicoletti, A. Cavalleri, Nonlinear light-matter interaction at terahertz frequencies. Adv. Opt. Photon. 8, 401-464 (2016)

15. H.A. Hafez, X. Chai, A. Ibrahim, S. Mondal, D. Férachou, X. Ropagnol, T. Ozaki, Intense terahertz radiation and their applications. J. Opt. 18, 093004 (2016)

16. D.M. Mittleman, Perspective: terahertz science and technology. J. Appl. Phys. 122, 230901 (2017)

17. S.W. Smye, J.M. Chamberlain, A.J. Fitzgerald, E. Berry, The interaction between terahertz radiation and biological tissue. Phys. Med. Biol. 46(9), R101-R112 (2001)

18. M. Tang, M. Zhang, L. Xia, Z. Yang, S. Yan, H. Wang, D. Wei, C. Du, H.-L. Cui, Detection of single-base mutation of DNA oligonucleotides with different lengths by terahertz attenuated total reflection microfluidic cell. Biomed. Opt. Express 11, 5362-5372 (2020)

19. M. Gupta, V. Savinov, N. Xu, L. Cong, G. Dayal, S. Wang, W. Zhang, N.I. Zheludev, R. Singh, Sharp toroidal resonances in planar terahertz metasurfaces. Adv. Mater. 28(37), 8206-8211 (2016)

20. A. Ahmadivand, B. Gerislioglu, N. Pala, Large-modulation-depth polarization-sensitive plasmonic toroidal terahertz metamaterial. IEEE Photon. Technol. Lett. 29(21), 1860-1863 (2017)

21. M. Shalaby, M. Peccianti, Y. Ozturk, R. Morandotti, A magnetic non-reciprocal isolator for broadband terahertz operation. Nat. Commun. 4(1558), 2572 (2013)

22. M. Shalaby, C. Vicario, C.P. Hauri, Simultaneous electronic and the magnetic excitation of a ferromagnet by intense $\mathrm{THz}$ pulses. New J. Phys. 18, 013019 (2016)

23. M. Shalaby, C. Vicario, K. Thirupugalmani, S. Brahadeeswaran, C.P. Hauri, Intense THz source based on BNA organic crystal pumped at Ti:sapphire wavelength. Opt. Lett. 41, 1777-1780 (2016)

24. H. Zhao, Y. Tan, T. Wu, G. Steinfeld, Y. Zhang, C. Zhang, L. Zhang, M. Shalaby, Efficient broadband terahertz generation from organic crystal BNA using near infrared pump. Appl. Phys. Lett. 114, 241101 (2019)

25. F. Roeder, M. Shalaby, B. Beleites, F. Ronneberger, A. Gopal, $\mathrm{THz}$ generation by optical rectification of intense near-infrared pulses in organic crystal BNA. Opt. Express 28, 36274-36285 (2020)

26. I.C. Tangen, G.A. Valdivia-Berroeta, L.K. Heki, Z.B. Zaccardi, E.W. Jackson, C.B. Bahr, D.J. Michaelis, J.A. Johnson, Comprehensive characterization of terahertz generation with the organic crystal BNA. J. Opt. Soc. Am. B 38, 2780-2785 (2021)

27. A.G. Stepanov, C. Ruchert, J. Levallois, C. Erny, C.P. Hauri, Generation of broadband $\mathrm{THz}$ pulses in organic crystal $\mathrm{OH} 1$ at room temperature and $10 \mathrm{~K}$. Opt. Mat. Express 4, 871-875 (2014)

28. Z.B. Zaccardi, I.C. Tangen, G.A. Valdivia-Berroeta, C.B. Bahr, K.C. Kenney, C. Rader, M.J. Lutz, B.P. Hunter, D.J. Michaelis, J.A. Johnson, Enabling high-power, broadband $\mathrm{THz}$ generation with 800-nm pump wavelength. ArXiv e-prints, 2010.02380 (2020)

29. C.A. Schmuttenmaer, Exploring dynamics in the far-infrared with terahertz spectroscopy. Chem. Rev. 104, 1759-1780 (2004)

30. S. Casalbuoni, B. Schmidt, P. Schmüser, V. Arsov, S. Wesch, Ultrabroadband terahertz source and beamline based on coherent transition radiation. Phys.Rev.ST.Accel.Beams12, 030705 (2009)

31. J. Neu, C.A. Schmuttenmaer, Tutorial: an introduction to terahertz time domain spectroscopy (THz-TDS). J. Appl. Phys. 124, 231101 (2018) 
32. J.P. Shan, A.S. Weling, E. Knoesel, L. Bartels, M. Bonn, A. Nahata, G.A. Reider, T.F. Heinz, Single-shot measurement of terahertz electromagnetic pulses by use of electro-optic sampling. Opt. Lett. 25, 426-428 (2000)

33. A. Gopal, S. Herzer, A. Schmidt, P. Singh, A. Reinhard, W. Ziegler, D. Brömmel, A. Karmakar, P. Gibbon, U. Dillner, T. May, H.-G. Meyer, G.G. Paulus, Observation of gigawatt-class THz pulses from a compact laser-driven particle accelerator. Phys. Rev. Lett. 111, 074802 (2013)

34. S. Herzer, A. Woldegeorgis, J. Polz, A. Reinhard, M. Almassarani, B. Beleites, F. Ronneberger, R. Grosse, G.G. Paulus, U. Hübner, T. May, A. Gopal, An investigation on THz yield from laser-produced solid density plasmas at relativistic laser intensities. New J. Phys. 20, 063019 (2018)

35. M. Sajadi, M. Wolf, T. Kampfrath, Terahertz-field-induced optical birefringence in common window and substrate materials. Opt. Express 23, 28985-28992 (2015)

36. A. Woldegeorgis, T. Kurihara, B. Beleites, J. Bossert, R. Grosse, G.G. Paulus, F. Ronneberger, A. Gopal, THz Induced Nonlinear Effects in Materials at Intensities above. J Infrared Milli Terahz Waves 39, 667-680 (2018)

37. V.E. Rogalin, I.A. Kaplunov, G.I. Kropotov, Opt. Spectrosc. 125, $1053(2018)$

38. F. D'Angelo, Z. Mics, M. Bonn, D. Turchinovich, Ultra-broadband $\mathrm{THz}$ time-domain spectroscopy of common polymers using THz air photonics. Opt. Express 22, 12475-12485 (2014)

39. http://www.tydexoptics.com/ru/products/thzl_optics/ / thz\_materials/

40. A. Gopal, T. May, S. Herzer, A. Reinhard, S. Minardi, M. Schubert, U. Dillner, B. Pradarutti, J. Polz, T. Gaumnitz, M.C. Kaluza, O. Jäckel, S. Riehemann, W. Ziegler, H. Gemuend, H. Meyer, G.G. Paulus, Observation of energetic terahertz pulses from relativistic solid density plasmas. New J. Phys. 14, 083012 (2012)

41. D.E. Aspnes, A.A. Studna, Dielectric functions and optical parameters of Si, Ge, GaP, GaAs, GaSb, InP, InAs, and InSb from 1.5 to 6.0 eV. Phys. Rev. B 27, 985-1009 (1983)

42. A.S. Barker, Transverse and Longitudinal Optic Mode Study in $\mathrm{MgF}_{2}$ and $\mathrm{ZnF}_{2}$. Phys. Rev. 136, A1290-A1295 (1964)

43. G. Gallot, J. Zhang, R.W. McGowan, T.-I. Jeon, D. Grischkowsky, Measurements of the $\mathrm{THz}$ absorption and dispersion of $\mathrm{ZnTe}$ and their relevance to the electro-optic detection of $\mathrm{THz}$ radiation. Appl. Phys. Lett. 74, 3450-3452 (1999)

44. Q. Wu, X.C. Zhang, 7 terahertz broadband GaP electro-optic sensor. Appl. Phys. Lett. 70, 1784-1786 (1997)

45. K. Walter, Solid-State Laser Engineering, 6th edn. (Springer, New York, 2006)

46. H.S. Carslaw, J.C. Jaeger, Conduction of Heat in Solids ((Oxford University Press, 1959), p. 189

47. X.C. Zhang, X.F. Ma, Y. Jin, T. Lu, E.P. Boden, P.D. Phelps, K.R. Stewart, C.P. Yakymyshyn, Terahertz optical rectification from a nonlinear organic crystal. Appl. Phys. Lett. 61, 3080-3082 (1992)
48. K. Kuroyanagi, M. Fujiwara, H. Hashimoto, H. Takahashi, S. Aoshima, Y. Tsuchiya, Determination of refractive indices and absorption coefficients of highly purified n-benzyl-2-methyl4-nitroaniline crystal in terahertz frequency regime. Jpn. J. Appl. Phys. 45, L761-L764 (2006)

49. K. Miyamoto, S. Ohno, M. Fujiwara, H. Minamide, H. Hashimoto, H. Ito, Optimized terahertz-wave generation using BNA-DFG. Opt. Exp. 17, 14832-14838 (2009)

50. T. Notake, M. Saito, M. Tang, Y.Y. Wang, H. Minamide, H. Ito, Solution growth of an organic N-benzyl-2-methyl-4-nitroaniline (BNA) crystal for DFG-THz source. 35th International Conference on Infrared, Millimeter, and Terahertz Waves, 1-2 (2010)

51. T. Notake, M. Takeda, S. Okada, T. Hosobata, Y. Yamagata, H. Minamide, Characterization of all second-order nonlinear-optical coefficients of organic N-benzyl-2-methyl-4-nitroaniline crystal. Sci. Rep. 9, 14853 (2019)

52. D. Kim, W.T. Kim, J. Han, J. Lee, S. Lee, B.J. Kang, M. Jazbinsek, W. Yoon, H. Yun, D. Kim, S.V. Bezouw, J. Campo, W. Wenseleers, F. Rotermund, O. Kwon, Wide-bandgap organic crystals: enhanced optical-to-terahertz nonlinear frequency conversion at near-infrared pumping. Adv. Opt. Mater. 8, 1902099 (2020)

53. M.C. Hoffmann, J.A. Fülöp, Intense ultrashort terahertz pulses: generation and applications. J. Phys. D: Appl. Phys. 44, 083001 (2011)

54. J.D. Wheeler, B. Koopman, P. Gallardo, P.R. Maloney, S. Brugge, G. Cortes-Medellin, R. Datta, C. Darren Dowell, J. Glenn, S. Golwala, C. McKenney, J.J. McMahon, Ch.D. Munson, M. Niemack, S.G. Parshley, G. Stacey, Millimeter, Submillimeter, and Far-Infrared Detectors and Instrumentation for Astronomy VII, Ed. by Holland W.S., Zmuidzinas J. Proc. SPIE. V. 9153. $91532 Z$ (2014)

55. E. Mavrona, F. Appugliese, J. Andberger, J. Keller, M. Franckié, G. Scalari, J. Faist, Terahertz refractive index matching solution. Opt. Express 27, 14536-14544 (2019)

56. V.V. Kubarev, Optical properties of CVD-diamond in terahertz range and its applications on the NovoFEL, 2007 Joint 32nd International Conference on Infrared and Millimeter Waves and the 15th International Conference on Terahertz Electronics, 863-865 (2007)

Publisher's Note Springer Nature remains neutral with regard to jurisdictional claims in published maps and institutional affiliations. 